\title{
Modified Chamberlain Procedure Is an Alternative Option with or Without VATS Still a Gold Standard Technique: A 10 Years Experience
}

\author{
Arun Kumar Haridas*, Bharathi Shridhar Bhat \\ Department of Cardiovascular and Thoracic Surgery, B K Shah Medical College, Vadodara, India \\ Email address: \\ arunkumarharidas@gmail.com (A. K. Haridas) \\ ${ }^{*}$ Corresponding author
}

To cite this article:

Arun Kumar Haridas, Bharathi Shridhar Bhat. Modified Chamberlain Procedure Is an Alternative Option with or Without VATS Still a Gold Standard Technique: A 10 Years Experience. International Journal of Cardiovascular and Thoracic Surgery. Vol. 4, No. 2, 2018 , pp. 14-19. doi: $10.11648 /$ j.ijcts.20180402.11

Received: May 8, 2018; Accepted: May 31, 2018; Published: June 14, 2018

\begin{abstract}
Introduction: Mediastinum is divided in anterior, middle and posterior part. Mediastinum contains heart, lung, thymus, lymph nodes and nerves. A varied spectrum of disease can arise from these organs. It poses a challenge to the clinicians to confirm the diagnosis. A Mediastinal lesion grows beyond the individual division of origin in advanced stages. It is the biopsy and histopathological study that gives us an idea about probable site or organ of origin and nature of the lesion. Diagnostic biopsy approach for such cases would be CT guide FNAC, Mediastinoscopy, Video Assisted Thoracoscopic Surgery, Chamberlain procedure and Thoracotomy. Anterior mediastinotomy known as chamberlain procedure is time tested technique for confirmation of the mediastinal lesions. Objective: To re-evaluate the technique of chamberlain procedure and its modifications to confirm the diagnosis of various lesions found in the mediastinum. Chamberlain procedure and other mediastinoscopic biopsy techniques have been replaced with VATS in most centres. In view of difficulties in gaining VATS instruments as they are expensive and its steep learning curve, we continue to do chamberlain procedure for mediastinal lesions biopsy. Method: We studied 75 patients, presented with various mediastinal mass or lung lesions. Most of these lesions were not identified from another mode of investigations. Chamberlain Procedure adopted to confirm the diagnosis, asses the stage of disease, operability status and to plan the treatment. Conclusion: Chamberlain procedure is still a gold standard over multiple other techniques in diagnosis and confirmation of the mediastinal diseases. It allows accessing any plane and location in the mediastinum. Result: Chamberlain procedure and biopsy can be done for diagnosis and confirmation of all kinds of mediastinal lesions. Compared to VATS, chamberlain procedure results and outcomes are similar.
\end{abstract}

Keywords: VATS, Chamberlain Procedure, Tuberculosis, Lung Cancer, Sarcoidosis, Thymus

\section{Introduction}

The mediastinum in the thorax extends from the thoracic inlet to the diaphragm and between the both pleural cavities. It contains vital structures of the circulatory, respiratory, digestive, thymic, lymphatic and nervous system. The mediastinum is further subdivided into three major areas, anterior, middle, and posterior compartments. The anterior mediastinum is a region posterior to the sternum and anterior to the heart pericardium and brachiocephalic vessels. It extends from the thoracic inlet to the diaphragm and contains the thymus gland, fat, and lymph nodes [1]. Anterior mediastinum divided into two parts, above the upper level of the pericardium named as superior mediastinum and below it as inferior mediastinum. Majority of the tumours arises from anterior mediastinum in adults.

The commonest lesions arising from the structures contained within the mediastinum are lymphoproliferative disorders, thymoma, thymic carcinoma, bronchopulmonary carcinoma, neurogenic tumours and germ cell tumours. Infective lesions like pulmonary and lymph node tuberculosis, inflammatory lesions due to sarcoidosis also 
found. Accurate diagnosis and staging of patients with cancer are imperative for an appropriate treatment strategy.

In majority of the cases it is difficult to confirm the diagnosis despite various appropriate investigations. Different approaches advocated to confirm the diagnosis based on location and organ from which lesion origins. Percutaneous CT guided FNAC, mediastinoscopic biopsy, VATS biopsy, chamberlain procedure and rarely thoracotomy biopsy are done to confirm the diagnosis. Majority of them have acceptable accuracy to diagnose the disease [2].

Chamberlain procedure is time tested, a minimal invasive surgery, performed by the thoracic surgeons. It provides excellent access to the antero-superior mediastinum and the lesions arising in that location. The Chamberlain procedure is done to do biopsy of lung lesions, lymph nodes or mediastinal masses located in antero-superior mediastinum [3].

Usage of Video assisted thoracoscopic surgery (VATS) and Robotic assisted thoracoscopic surgery (RATS) are wide spread in western countries, lead to decreased significance of chamberlain and other mediastinal tissue biopsy techniques. We have reviewed experience of chamberlain procedure and its modifications to diagnose various mediastinal mass, lymph node and lung lesions.

\section{Method}

\subsection{Material}

Seventy-five patients $(\mathrm{n}=75)$, had under gone chamberlain procedure and its modified techniques between December 2007 to December 2017 were reviewed. Cases were operated and followed by the single surgeon. Details of a patient's history, investigation and surgical outcome were evaluated.

\subsection{Method}

All cases were operated under general anaesthesia. Single lung ventilation was used with DLT (double lumen ET tube) for better exposure of lesions and vascular structures.

Patients operated in supine position. About $4 \mathrm{~cm}$ incision taken over the superior border of second rib on selected side of chest, just lateral to the sternum. Muscle layer divided and internal mammary artery (IMA) was ligated. Pleura opened electively for the lung, hilar nodes biopsy. (Figure-1, 2)

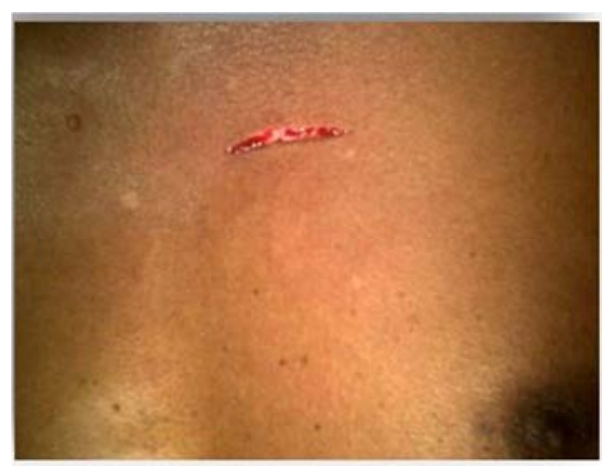

Figure 1. Left thoracic incision.

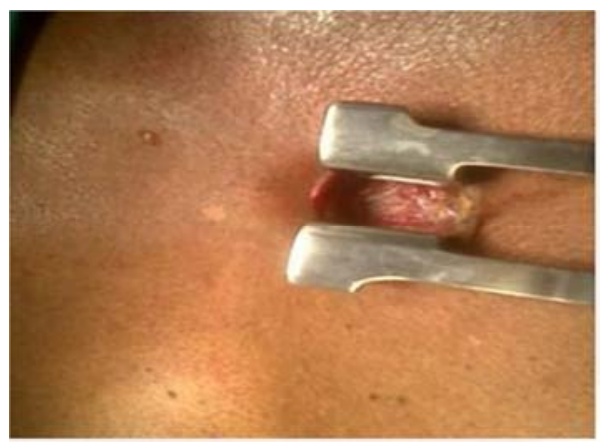

Figure 2. Dissection 2nd ICS.

In most cases no intercostal drainage tube (ICD) used. Wound closed in layers under full expansion of lung with help of an anaesthetist. ICD was inserted only in cases of air leak from the lung biopsy site and expecting likely bleeding from the lesions. (Figure-3, 4).

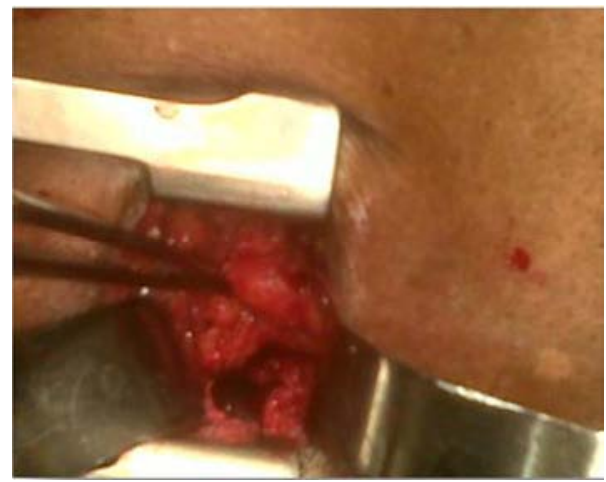

Figure 3. Mediastinal dissection.

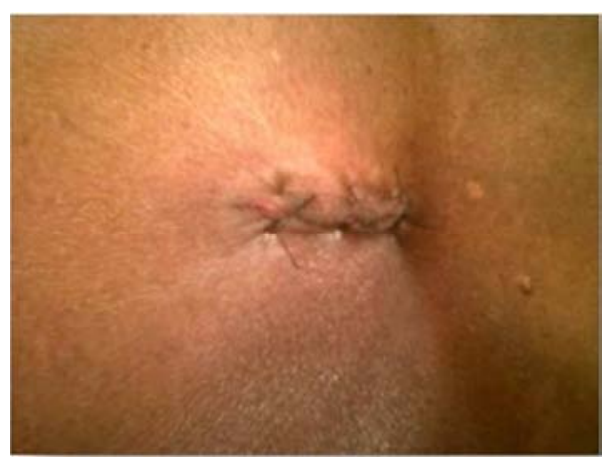

Figure 4. Closed incision.

Patients were extubated on the table. Most patients were discharged by evening on the same day. (Figure $-5,6$ ).

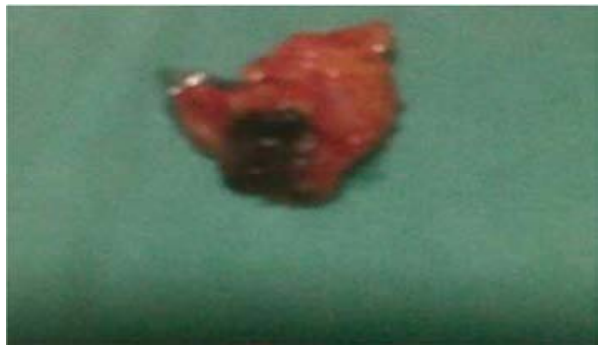

Figure 5. Specimen lymph node. 


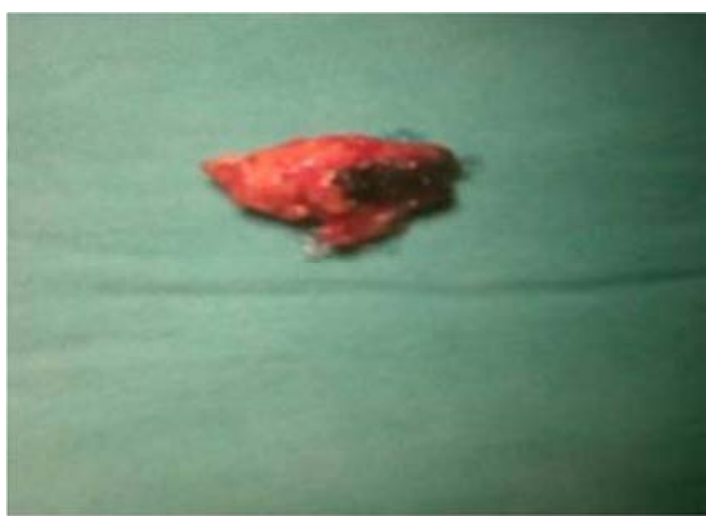

Figure 6. Specimen lymph node.

\section{Statistical Analysis}

The statistical software used for statistical analysis is SPSS 20.

\section{Results}

It is a retrospective study. Total seventy-five $(n=75)$ patients were reviewed. It comprised of Forty-seven male $(62.67 \%)$ and 28 female (37.33\%) population. The mean age was 45.18 years (SD-16.61) and 48.26 years (SD-1.35) for female and male respectively.

Majority of patients presented with chronic cough along with or without breathlessness, fever and weight loss. Patients suspected of tuberculosis had cough, low grade fever and weight loss. Thirty-four (45.33\%) patients had a chronic cough at the time of presentation. (Figure-7)

Chest $\mathrm{x}$-ray was found to be poor investigative tool to diagnose majority of the mediastinal masses and lung lesions. It could reveal lesions, only in twenty patients $(26.67 \%)$. Computed Tomography (CT) chest scan is the pathognomic test revealing the lesions with $100 \%$ accuracy.

Out of 75 patients, twenty lymph node $(26.67 \%)$, twentyfour mediastinal mass (32\%) and twenty-one lung lesion $(28.0 \%)$ biopsy done. (Figure- 8 )

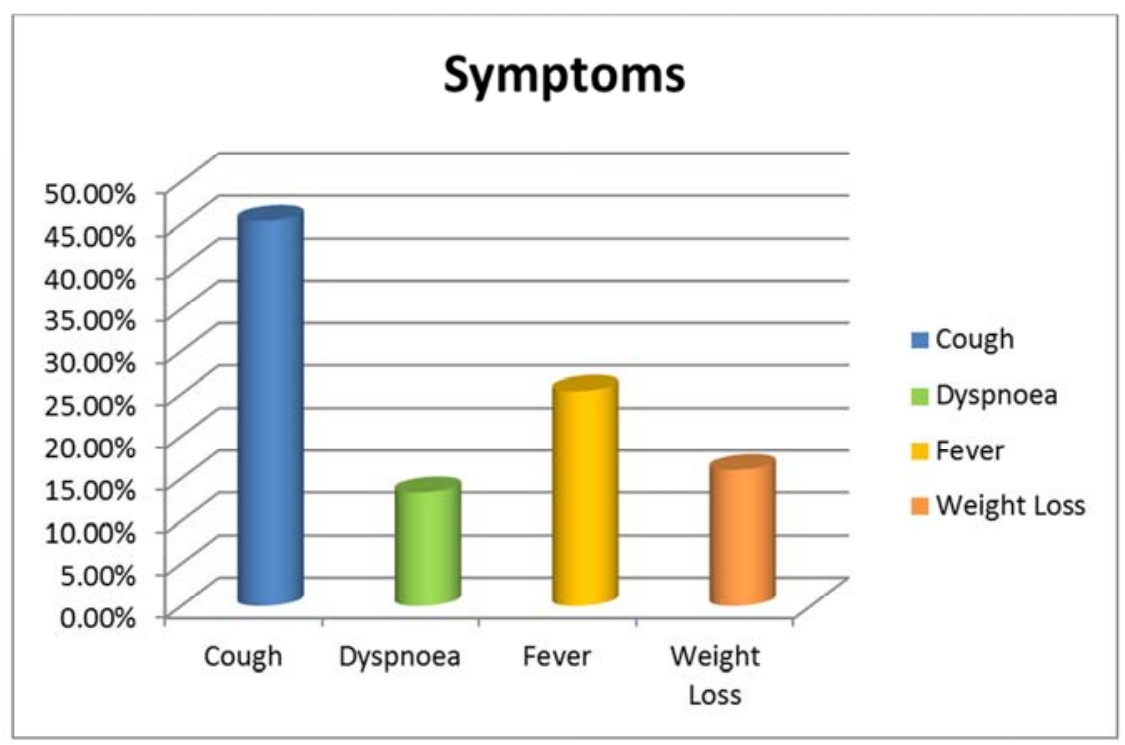

Figure 7. Presenting symptoms.

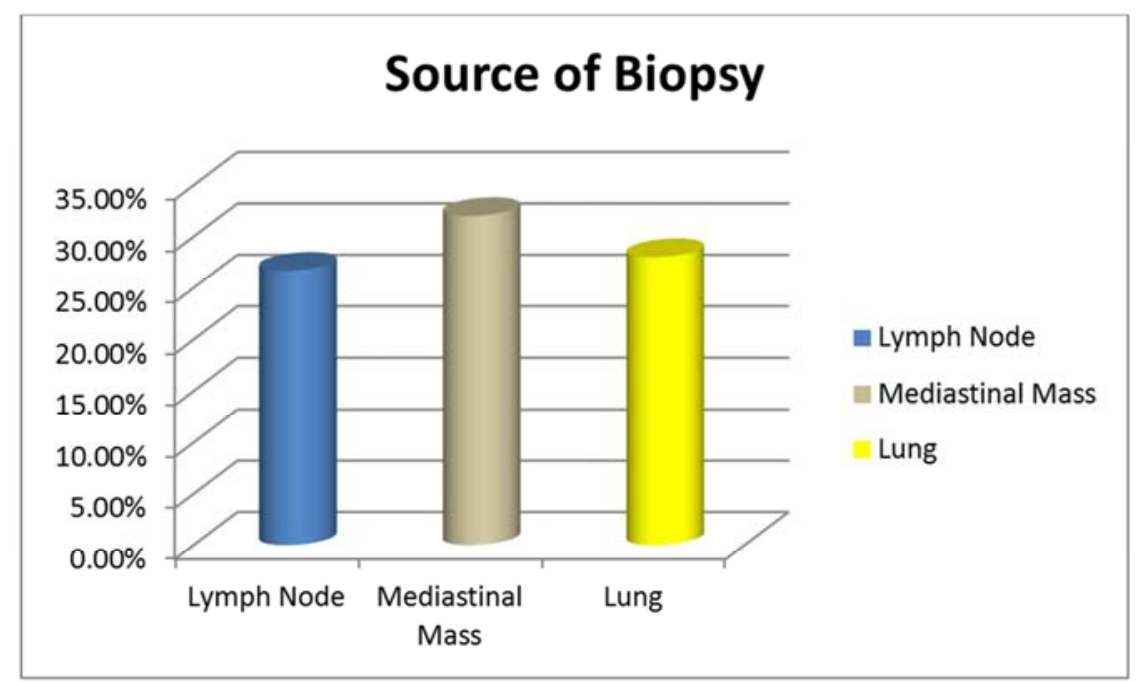

Figure 8. Source of biopsy. 
Histopathological examination revealed following data. Out of 75 cases, 26 tuberculosis $(34.67 \%), 18$ lymphoma $(24 \%), 12$ thymic tumours $(16 \%)$, eight bronchogenic tumours $(10.67 \%)$, four sarcoidosis $(5.33 \%)$, four bacterial infection $(5.33 \%)$, two benign hyperplasia of lymph node $(2.67 \%)$ and one fungal infection (1.33\%). (Figure-9)

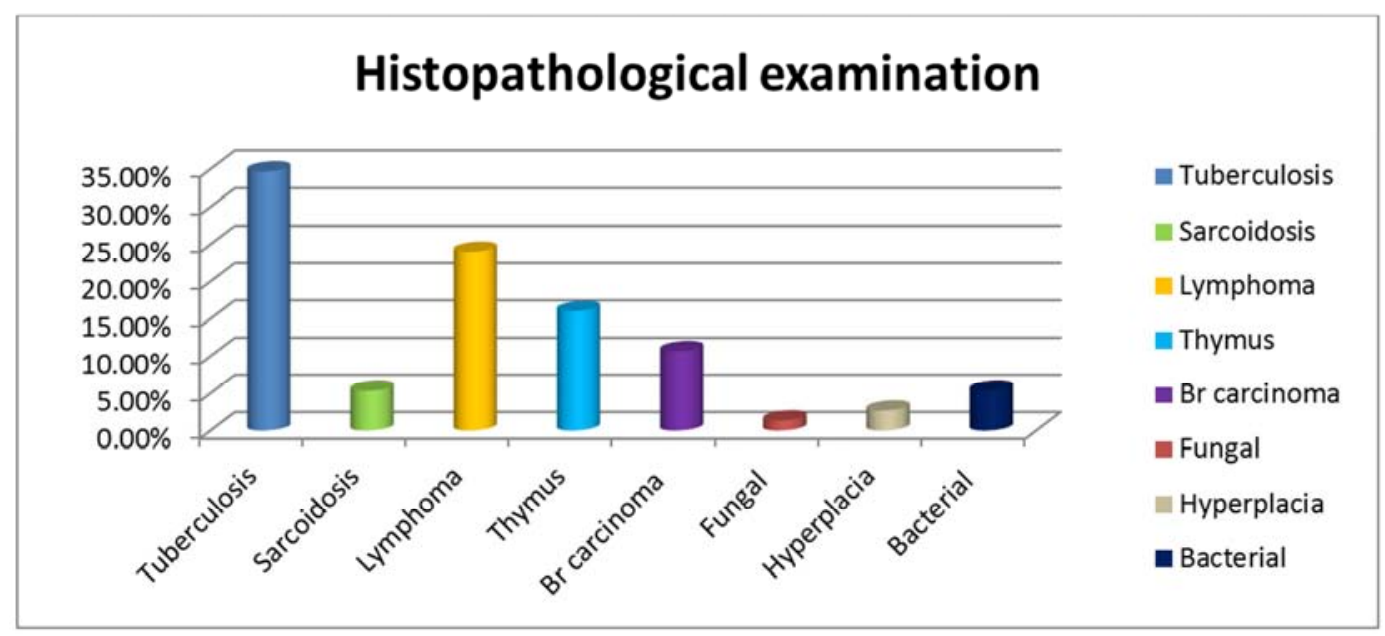

Figure 9. Histopathological examination.

We encountered minor complications in eight cases (10.67\%). Most were self limiting. Six patients (8\%) had minor bleeding, controlled with packing. One case $(1.33 \%)$ had pneumothorax while doing extra pleural, mediastinal mass biopsy. It was revealed in post-op chest X-ray and treated with ICD insertion. One lung biopsy case had $(1.33 \%)$ minor air leak treated by keeping ICD for an extra one day (Figure-10).

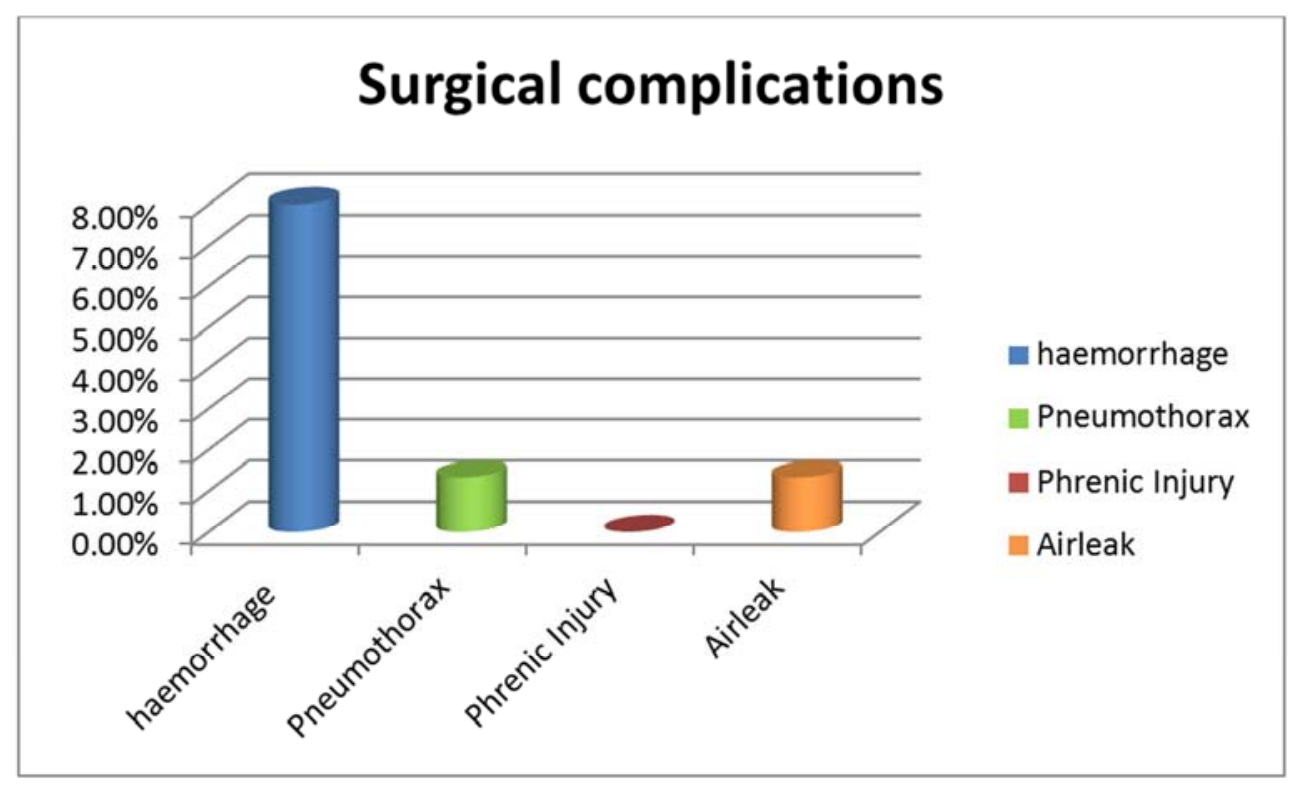

Figure 10. Surgical complications.

Mean time of 36.59 minutes (SD-5.81) taken to complete most procedures. In three cases $(4 \%)$ incision was extended for one or two centimetres to improve the exposure of tissues and to safe guard the vascular structures.

\section{Discussions}

Mediastinum contains all vital organs of the body. It can give rise to various abnormal lesions. The lesions can be due to tumours, infection and inflammatory reactions [4].
Commonest location of lesions is anterior mediastinum in adults and posterior mediastinum in children. Thymic tumours and lymphomas are frequent in adults and neurogenic tumours in children. Most malignant tumours are found in adults compared to children $[5,6]$.

The biopsy technique depends on location of the lesion in the mediastinum. Biopsies are done for diagnostic and therapeutic purposes. One standard approach of biopsy is not appropriate due to the wide spectrum of diseases [7]. Diagnostic confirmations of mediastinal lesions are crucial to 
stage and plan the treatment. So biopsy is imperative to clinch the diagnosis.

Diagnosis of pathological lesions affecting the lungs and mediastinum was challenging. Various techniques devised over a period as per the need and location of lesions. To mention a few, Daniel's biopsy of the scalene pad of fat, Harken cervicomediastinal explorative biopsy, Steele cervical mediastinotomy and biopsy, Carlens mediatinoscopic biopsy. Most common procedures used till dates are Anderson transbronchoscopic lung and nodes biopsy, Chamberlain anterior mediastinotomy and biopsy, CT-guided needle FNAC and biopsy.

Cervical mediastinoscopy was first described in 1959 by Carlens from Sweden, later popularized by Pearson in the early 1960s. This procedure gives access to the middle mediastinum along the pre and paratracheal areas down to the tracheal bifurcation. In 1971 Kirschner modified the classical approach to the extended approach to do the lung biopsy.

Cervical mediastinoscopy is used, to do the various node biopsies to confirm and stage the cancers. It can also diagnose granulomatous lesions [8]. Extended mediastinoscopy helps to explore enlarged anterior mediastinal, subaortic window lymph nodes and upper lobe of lung lesions [9]. Major complications are rarely encountered [10]. The commonest vessels injured are innominate and azygous veins [11]. Minor bleeding is treated by packing but the major bleeding needs conversion in to thoracotomy [12].

In 1965 Chamberlain described the technique of anterior mediastinotomy. It had more advantage compared to other techniques. This procedure can provide a histological diagnosis where none other techniques could confirm the diagnosis [13]. It not only confirms the diagnosis but also helps to detect unresectable diseases. Evaluate whether the operation required eliminating the local disease is tolerated by the patients or not. This procedure does not interfere with performing a formal subsequent thoracotomy [14]

Interpreting CT guided fine needle aspiration biopsy (FNAB) from the mediastinum is challenging. Majority of the times specimen may not be adequate to give conclusive diagnosis. It needs high accuracy and experience to enter the lesion as a likely chance of injury to the vascular structures is high. Studies have shown a wide range of a sensitivity and specificity of $13 \%$ to $88 \%$ [15].

Popularity of Video assisted thoracoscopic surgery (VATS) and Robotic assisted thoracoscopic surgery (RATS) lead to a decline in the usage of mediastinal biopsy techniques. They are perceived as less invasive techniques [16]. VATS is widely used by most thoracic surgeon in western countries. It is advocated for lung, lymph node and mediastinal mass biopsy. It is proven safe, reliable technique for diagnostic and therapeutic purposes. It can offer high diagnostic accuracy due to large tissue samples [17]. Though diagnostic efficacy of VATS is evident but cost of treatment is high and it needs steep learning curve [18].

VATS is well suited for diagnosis of pleural and pulmonary diseases. Biopsies of malignant tumours and total excisions of benign lesions by VATS are less morbid than by an open thoracotomy. The use of VATS for the diagnosis and excision of benign tumours is accepted however for resection of malignant tumours is not accepted universally [19]. Majority of studies based on VATS have shown no extra benefits. Operating time, stay in hospital, post-op pain and complications remained similar compared to an open technique [20].

Most tumours in the mediastinum are accessible for biopsy and total resection with acceptable complications by VATS $[21,22]$. There are reports showing a higher incidence of minor complications in VATS compared to open thoracotomy [23]. Major vascular injuries are rare, which needs conversion in to thoracotomy to control the bleeding. Most important drawback of VATS is, cannot palpate the lesions and verify the tissue consistency [24].

Chamberlain procedure is a great technique at harvesting lymph nodes and biopsying mediastinal mass [25]. General anesthesia is preferred for the procedure. Patients with severe orthopnea, morbid obesity and concomitant asthma need extra precautions [26]. We did all cases under general anaesthesia with single lung ventilation, which permitted wider access to mediastinum and to safeguard the vascular structures. We modified the technique, by changing the location of incision based on the lesions. These modifications improved our success rate of retrieving biopsy. Average operating time was as comparable to VATS [27]. A combined approach of cervical mediastinoscopy and chamberlain procedure, to assess preoperative staging and resectability of tumour is advocated [28].

The mediastinum is the most common site for the primary intrathoracic pathology in children. Chamberlain procedure and biopsy is a versatile technique with diagnostic accuracy $100 \%$ in such groups [29].

Chamberlain procedure gives accurate information by inspection, palpation and biopsies of lymph nodes or tumours affecting the mediastinum. Similar to VATS, it gives access to lymph nodal stations 5 and 6 [30]. It reveals direct invasion of the mediastinum from adjacent tumours. Chamberlain procedure is a simple, safe and formidable procedure can be adapted to specific diagnostic and therapeutic purposes [31]. It retrieves large biopsy tissue, so enhances the overall report positivity and gives better control over procedure related complications. [32]

\section{Conclusion}

Chamberlain procedure is a versatile surgical technique, gives access to the mediastinum and its structures. It allows tissue palpation to confirm the specimen. Single lung ventilation, surgical loupe with appropriate magnification and head light would make procedure easy, safe, faster and cost effective compared to VATS. It does not require any learning curve. It is suitable for all age groups. It is a day care procedure. Chamberlain procedure is still the gold standard technique and can be utilized alternative to VATS. 


\section{References}

[1] Biondi A, Rausei S, Cananzi FC, Zoccali M, D'Ugo S, Persiani R. Surgical anatomy of the anterior mediastinum. Ann Ital Chir. 2007; 78(5):351-3.

[2] Assaad MW, Pantanowitz L, Otis CN. Diagnostic accuracy of image-guided percutaneous fine needle aspiration biopsy of the mediastinum. Diagn Cytopathol. 2007; 35(11):705-9.

[3] Olak. J. Parasternal mediastinotomy (Chamberlain procedure). Chest Surg Clin N Am. 1996; 6(1):31-40.

[4] Korst R. J. Mediastinal Biopsy Techniques. Mediastinal Lesions. Springer, Cham. 2017. pp 5-24 DOI. https://doi.org/10.1007/978-3-319-48379-5_2

[5] Tetsuro Araki, Mizuki Nishino, Wei Gao, Josee Dupuis, George R, Washko Gary M, et al. Anterior mediastinal masses in the Framingham Heart Study: Prevalence and CT image characteristics. European Journal of Radiology. 2015; 2:26-31.

[6] Takeda S, Miyoshi S, Akashi A, Ohta M, Minami M, Okumura M, Masaoka A, Matsuda H. Clinical spectrum of primary mediastinal tumors: a comparison of adult and pediatric populations at a single Japanese institution. J Surg Oncol. 2003 May; 83(1):24-30.

[7] Brett W. Carter, Edith M. Marom, Frank C, Detterbeck. Approaching the Patient with an Anterior Mediastinal Mass: A Guide for Clinicians. J Thorac Oncol. 2014; 9: 102-109.

[8] Kirschner P. Cerival Mediatinoscopy. Chest Surg Clincs of North America. 1996; 6(1) 1-20.

[9] Ginsberg, Robert. Extended Cervical Mediatinoscopy. Chest Surg Clincs of North America. 1996; 6(1) 21-30.

[10] Heikki J, Puhakka. Complications of mediastinoscopy. J Laryng Otol, 1989; 103:312-315.

[11] Urschel J. Conservative Management (Packing) of Hemorrhage Complicating Mediatinoscopy. Ann Thorac Cardiovasc Surg, 2000; 6(1):9-12.

[12] Park JB. Management of major hemorrhage during mediastinoscopy, J Thorac Cardiovasc Surg 2003: 126; 726-31.

[13] Shimizu J, Kamesui T, Moriya M, Murata S, Nakanishi I, Sasaki M, Minato H. Four cases of invasive anterior mediastinal tumors definitively diagnosed by the chamberlain procedure. Ann Thorac Cardiovasc Surg. 2014; 20 Suppl: 434-40. Doi: 10.5761/atcs.cr.12.02185.

[14] McNeill TM, Chamberlain JM. Diagnostic anterior mediastinotomy. Ann. Thorac. Surg. 1966; 2:532.

[15] Powers CN, Silverman JF, Geisinger KR, Frable WJ. Fineneedle aspiration biopsy of the mediastinum. A multiinstitutional analysis. Am J Clin Pathol. 1996; 105(2):168-73.

[16] Krishna S Vyas, Daniel L Davenport, Victor A, Ferraris, Saha, Mediastinoscopy: Trends and Practice Patterns in the United StatesSouth Med J. 2013 Oct; 106(10): 539-544.

[17] Federico Tacconi, Paola Rogliani, Benedetto Cristino, Francesco Gilardi, Leonardo Palombi, Eugenio Pompeo. Minimalist video-assisted thoracic surgery biopsy of mediastinal tumors. J Thorac Dis. 2016; 8(12): 3704-3710. doi:10.21037/jtd.2016.06.33
[18] Zakkar M, Hunt I. Complication rates in mediastinoscopy and training: video versus conventional mediastinoscopy. Ann Thorac Surg. 2012; 94:337.

[19] Demmy, Todd L et al. Multicenter VATS experience with mediastinal tumorsThe Annals of Thoracic Surgery. 1998; 66(1):187-192.

[20] Molin LJ, Steinberg JB, Lanza LA. VATS increases costs in patients undergoing lung biopsy for interstitial lung disease. Ann Thorac Surg. 1994 Dec; 58(6):1595-8.

[21] Landreneau RJ, Dowling RD, Castillo WM, Ferson PF. Thoracoscopic resection of an anterior mediastinal tumor. Ann Thorac Surg. 1992; 54: 142-144.

[22] Sugarbaker DJ. Thoracoscopy in the management of anterior mediastinal masses. Ann Thorac Surg. 1993; 56: 653-656.

[23] Zakkar M, Tan C, Hunt I. Is video mediastinoscopy a safer and more effective procedure than conventional mediastinoscopy? Interact Cardiovasc Thorac Surg. 2012; 14:81-84.

[24] Sameer A, Hirji Scott S, Balderson, Mark F, Berry, Thomas A, D'Amico. Troubleshooting thoracoscopic anterior mediastinal surgery: lessons learned from thoracoscopic lobectomy. Ann Cardiothorac Surg. 2015; 4(6): 545-549. doi: 10.3978/j.issn.2225-319X.2015.07.04.20.

[25] Nechala P, Graham AJ, McFadden SD, Grondin SC, Gelfand G. Retrospective analysis of the clinical performance of anterior mediastinotomy. Ann Thorac Surg. 2006; 82(6):20049.

[26] Alireza Sharifian Attar, Reza Jalaeian Taghaddomi, Reza Bagheri. Anesthetic Management of Patients With Anterior Mediastinal Masses Undergoing Chamberlain Procedure (Anterior Mediastinotomy). Iran Red Crescent Med J. 2013; 15(4): 373-374. doi:10.5812/ircmj.2120

[27] Vincenzo Ambrogi, Tommaso Claudio Mineo. VATS biopsy for undetermined interstitial lung disease under non-general anesthesia: comparison between uniportal approach under intercostal block vs. three-ports in epidural anesthesia. J Thorac Dis. 2014 Jul; 6(7): 888-895. doi: 10.3978/j.issn.20721439.2014.07.06

[28] Jiao X, Magistrelli P, Goldstraw P. The value of cervical mediastinoscopy combined with anterior mediastinotomy in the peroperative evaluation of bronchogenic carcinoma of the left upper lobe. Eur J Cardiothorac Surg. 1997; 11(3):450-4.

[29] Salas Valverde S, Gamboa Y, Vega S, Barrantes M, Gonzalez $\mathrm{M}$, Zamora JB. Diagnosis of anterior mediastinal mass lesions using the Chamberlain procedure in children. Pediatr Surg Int. 2008; 24(8):935-7. doi: 10.1007/s00383-008-2179-8.19

[30] Rami Porta R. Surgical exploration of the mediastinum by mediastinoscopy, parasternal mediastinotomy and remediastinoscopy: indications, technique and complications. Ann Ital Chir. 1999; 70(6):867-72.

[31] Fernadez A, Campos JR, Filomeno LT, Jatene FB. Mediastinoscopy: technical aspects and current indications. Rev Hosp Clin Fac Med Sao Paulo. 1994; 49(4):164-7.

[32] Hashim SW, Baue AE, Geha AS. The role of mediastinoscopy and mediastinotomy in lung cancer. Clin Chest Med. 1982; 3(2):353-9. 